\title{
Phase Coherence in Multiple Pulse Optical Spectroscopy
}

\author{
WARREN S. WARREN \\ Department of Chemistry, Princeton University, Princeton, NJ 08544
}

\author{
AHMED H. ZEWAIL $\dagger$ \\ Arthur Amos Noyes Laboratory of Chemical Physics, $\$$ California Institute of \\ Technology, Pasadena, CA 91125
}

In this paper we describe a new technique for the generation of multiple pulse phase coherent sequences in optical spectroscopy. The technique is an extension of the acousto-optic modulation and fluorescence detection methods developed for optical transitions by Zewail and Orlowski (Zewail et al., Chem. Phys. Lett. 48, 256 (1977); Orlowski et al., Chem. Phys. Lett. 54, 197 (1978)). Application of these multiple pulse trains (of different phases) to optical transitions of two-level and multilevel systems is demonstrated experimentally. It is shown that they can be used to (i) suppress spontaneous emission background, (ii) enhance coherent transients such as photon echoes, (iii) measure additional relaxation parameters in systems with complex rotationalvibrational levels, and (iv) enhance the effective laser bandwidths through composite pulse trains, as demonstrated on $I_{2}$ gas. Finally, the potential of this development is extended to the possibility of observing selective multiquantum excitation in molecules.

\section{INTRODUCTION}

A molecule which is irradiated with intense coherent pulses (for example, from a laser) can absorb energy and then coherently reradiate it. The quantitative effects of such pulses on pure two-level systems were first understoood in nuclear magnetic resonance: the classical picture of a magnetic field exerting a torque $\boldsymbol{\mu} \times \mathbf{B}$ on a magnetic

\footnotetext{
† Camille and Henry Dreyfus Foundation Teacher-Scholar.

$\ddagger$ Contribution No. 6803 .
} 
dipole is also basically correct quantum mechanically. ${ }^{1-4}$ Thus, a resonant magnetic field pulse can flip spins from their initial alignment, or create a new alignment in any other direction. This new alignment oscillates, which gives the exact transition frequency $\omega$; its perpendicular component decays, which gives the transverse relaxation (or coherence dephasing) time $T_{2}$; and finally the original alignment is restored, at a rate $T_{1}$ which is frequently not equal to $T_{2}$.

This simple classical picture cannot be directly extended to the optical regime. However, all possible two-level systems are mathematically quite similar. In fact, electronic or vibrational transitions can be treated (in the semiclassical limit)) as fictitious "spin flips," $, 5,6$ and laser pulse sequences analogous to those derived for NMR have been used to measure $T_{1}, T_{2}$ and the transition dipole moment $|\boldsymbol{\mu}|$ for these transitions. These parameters are in principle sufficient to completely characterize any two-level system, so simple laser pulse sequences have frequently been used to study energy transfer, ${ }^{7-9}$ collision effects, ${ }^{10-12}$ and laser propagation in optically dense media. ${ }^{13-15}$

In practice, however, the implicit assumption of only two isolated levels must be considered to be only a first approximation. Even an isolated large molecule has a high density of vibronic states plus relaxation mechanisms such as spontaneous emission which couple to nonresonant eigenstates. Intermolecular and intramolecular interactions further complicate the problem. These additional interactions would give very useful information if their effects could be interpreted; for example, the scalar spin-spin coupling in NMR splits all transitions into multiplets which reveal the molecular configuration. Unfortunately, in electronic spectroscopy there are usually so many distinct interactions (or so many coupled sites) that the individual contributions cannot be extracted. Simple pulse sequences (which still only measure $T_{1}, T_{2}, \omega$ and $|\boldsymbol{\mu}|$ ) reveal very little about these couplings, and have been previously noted as sometimes leading to physically unreasonable conclusions.

The most powerful technique for overcoming this spectral complexity and extracting additional information from strongly coupled spin systems is multiple pulse spectroscopy. To name only a few applications, pulse trains have been designed to eliminate the direct dipole-dipole or quadrupole interaction to measure chemical shift tensors orders of magnitude smaller, ${ }^{16,17}$ pump forbidden transi- 
tions ${ }^{18-22}$ (sometimes with high intensity), ${ }^{23-26}$ or resolve different spin interactions via two-dimensional Fourier transformation. ${ }^{27,28}$ In fact, these multiple pulse techniques and the advanced state of r.f.microwave technology are probably the most important reasons why NMR is one of the most powerful analytical techniques available today.

We have shown in recent papers ${ }^{29-33}$ that the strong mathematical similarities between coherent effects in NMR or optical spectroscopy imply that laser analogs of these multiple pulse sequences will be extremely valuable as probes of energy transport, dephasing mechanisms, and collisional dynamics. We have also shown ${ }^{29,30}$ that the acousto-optic modulation and fluorescence detection technique of Zewail and Orlowski ${ }^{7}$ can conveniently create phase coherent pulse trains, and we have used this technique to study gas phase dynamics. In this paper we summarize these results, comment on some extensions, and discuss new experimental developments.

\section{MULTIPLE PULSE TRAINS IN MULTILEVEL SYSTEMS-SחIID STATE APPLICATIONS}

The various strong electronic interactions in low temperature pure or mixed molecular crystals frequently make absorption lineshapes broad and featureless. Even if each individual molecule in the crystal has only one nearly resonant transition (ground state $G \rightarrow$ excited state $E$ ) a fairly comprehensive list of line broadening effects would include:

1) Long range inhomogeneous broadening, caused for example by crystal strains or surface effects; on the average, molecules which are close together have strongly correlated resonance frequencies.

2) Short range inhomogeneous broadening, caused for example by isotopic impurities randomly distributed throughout the sample. Resonance frequency is then independent of position.

Typical inhomogeneous linewidths are $0.1-1 \mathrm{~cm}^{-1}$, with the relative extents of (1) and (2) currently a subject of debate. The Hamiltonian for inhomogeneous broadening can be written as

$$
\mathscr{H}=\hbar \sum_{i} \omega_{i} \sigma_{z i}
$$


3) Exchange or transition multipole interactions between pairs of sites can be written as

$$
\begin{aligned}
\mathscr{H} & =\hbar \sum_{i>j} V_{i j}\left(\sigma_{i}^{+} \sigma_{j}^{-}+\sigma_{i}^{-} \sigma_{j}^{+}\right) \\
& =\hbar \sum_{i>j} 2 V_{i j}\left(\sigma_{x i} \sigma_{x j}+\sigma_{y i} \sigma_{y i}\right)
\end{aligned}
$$

where $V_{i j}$ for adjacent molecules can be several wavenumbers from exchange alone. For a dilute mixed crystal the contribution from transition dipole moments alone would generate a Lorentzian lineshape, with $T_{2}^{-1} \sim 1.5 f \mu^{2} a^{-3} \hbar^{-1}$ where $f$ is the fractional concentration and $a^{3}$ is the volume of the unit cell. ${ }^{31}$

4) Crystal stabilization energy terms or permanent multipole interactions can be written as

$$
\mathscr{H}=\hbar \sum Q_{i j} \sigma_{z i} \sigma_{z j}
$$

For a dilute mixed crystal the permanent dipole moments alone would also generate a Lorentzian lineshape, with $T_{2}^{-1} \sim 0.7 f(\Delta \mu)^{2} a^{-3} \hbar^{-1}$ where $\Delta \boldsymbol{\mu}$ is the change in dipole moment upon excitation. ${ }^{32}$

Calculating the expected lineshape when several different interactions are present is much more complex. Fortunately, the full Hamiltonian of Eqs. (1), (3), and (4) can be quite generally rewritten as:

$$
\begin{aligned}
\mathscr{H}= & \hbar\left(\Delta \omega(\mathbf{r}) \sigma_{z}+\sum_{i} \omega_{i} \sigma_{z i}+\sum_{i>j} D_{i j}\left(3 \sigma_{z i} \cdot \sigma_{z j}-\boldsymbol{\sigma}_{i} \cdot \boldsymbol{\sigma}_{j}\right)\right. \\
& \left.+\sum_{i>j} J_{i j}\left(\boldsymbol{\sigma}_{i} \cdot \boldsymbol{\sigma}_{j}\right)\right) \\
D_{i j}= & -\frac{2}{3} V_{i j}+\frac{1}{3} Q_{i j} \quad J_{i j}=\frac{4}{3} V_{i j}+\frac{1}{3} Q_{i j}
\end{aligned}
$$

which is identical to the NMR Hamiltonian for protons in a solid ${ }^{3}$ or a liquid crystal. ${ }^{34}$ Thus only minor modifications reflecting differences in the initial equilibrium distributions are required to transfer wellknown NMR results to the optical regime. Further details are contained in Refs. 32 and 33. We summarize these results in Table I.

In general, short-range broadening will produce distorted lineshapes in photon echo experiments. It may even generate strange lineshapes in simple absorption experiments. For example, Figure 1 shows an experimental spectrum ${ }^{35,36}$ and a Monte Carlo computer 
TABLE I

Effects of different line broadening mechanisms

\begin{tabular}{lcccc}
\hline & $\begin{array}{c}\text { Photon } \\
\text { echo }\end{array}$ & $\begin{array}{c}\text { Multiple echo } \\
\text { (Carr-Purcell) }\end{array}$ & $\begin{array}{c}\text { Dipolar line } \\
\text { narrowing }\end{array}$ & $\begin{array}{c}\text { Full line } \\
\text { narrowing }\end{array}$ \\
\hline $\begin{array}{l}\text { Long-range broadening } \\
\quad \omega \omega(\mathbf{r})\end{array}$ & - & - & + & - \\
$\begin{array}{l}\text { Short-range broadening } \\
\left(\omega_{i}\right)\end{array}$ & $\mathrm{D}$ & - & $\omega_{i} / \sqrt{3}$ & - \\
$\begin{array}{c}\text { Exchange, transition } \\
\text { multipole }\left(V_{i j}\right)\end{array}$ & $\mathrm{D}$ & $V_{i j}$ & $\frac{2}{3} V_{i j}$ & - \\
$\begin{array}{l}\text { Crystal stabilization, } \\
\text { Permanent multipole }\left(Q_{i j}\right)\end{array}$ & $\mathrm{D}$ & $Q_{i j}$ & $\frac{1}{3} Q_{i j}$ & - \\
$\begin{array}{l}\text { Spontaneous emission, } \\
\quad \text { relaxation mechanisms }\end{array}$ & + & + & & + \\
\hline
\end{tabular}

$+\equiv$ Contributes to total lineshape by simple convolution.

$\mathrm{D} \equiv$ Distorted contribution to total lineshape.

- $\equiv$ Does not contribute to lineshape.

simulation $^{32}$ for 1,4-dibromonaphthalene. This molecule has a strong exchange coupling $\left(V=-6.2 \mathrm{~cm}^{-1}\right)$ for adjacent molecules in one direction, $V \approx 0$ in all other directions, and short-range broadening from the $10 \%$ natural abundance of the mono- ${ }^{13} \mathrm{C}$ species, which is shifted by about $2 \mathrm{~cm}^{-1}$. In extreme cases $\left(\omega_{i}-\omega_{j} \gg V_{i j}\right)$ the short range broadening quenches exchange or transition dipole interactions. However, it can never quench permanent dipole interactions since $\left[Q_{i j} \sigma_{z i} \sigma_{z j}, \omega_{i} \sigma_{z i}\right]=0$.

Multiple pulse trains can separate out the different broadening mechanisms without distortions. Figures $2 a$ and $2 b$ are closely related to pulse trains which are commonly used in NMR. The middle of Figure $2 \mathrm{a}$ is a Carr-Purcell sequence. ${ }^{37}$ This sequence of its later modifications will precisely remove the linear operators in Eq. (5), since $\sigma_{z i} \leftrightarrow-\sigma_{z i}$ by the $180^{\circ}$ rotations. The bilinear operators $\sigma_{z i} \sigma_{z j}$ or $\boldsymbol{\sigma}_{i} \cdot \boldsymbol{\sigma}_{j}$ are unaffected. One version of the Carr-Purcell sequence has in fact been demonstrated in the infrared. ${ }^{38}$

The repeated unit of Figure $2 b$ is called a WAHUHA sequence ${ }^{17}$ and will exactly eliminate the $D_{i j}$ terms in Eq. (5), scale down the inhomogeneous broadening by $1 / \sqrt{3}$, and leave $J_{i j}=\frac{4}{3} V_{i j}+\frac{1}{3} Q_{i j}$ 


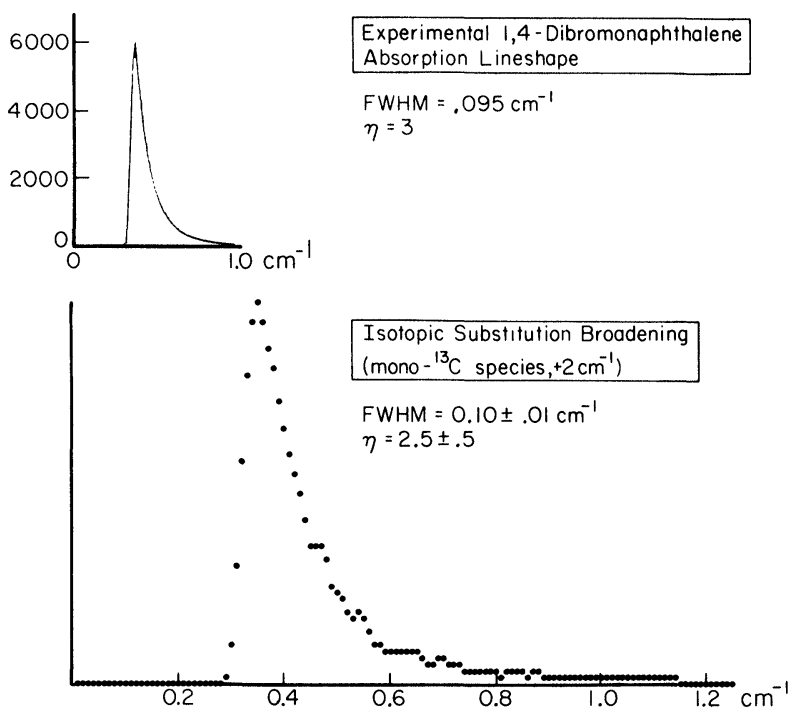

FIGURE 1 Experimental absorption lineshape of DBN at $4952 \AA$ (courtesy of $\mathrm{H}$. Port, University of Stuttgart) and our Monte Carlo simulation based on natural abundance carbon-13. The unusual lineshape is produced by cross terms between exchange coupling and inhomogeneous broadening.

unaffected. A combination of these two sequences (Figure 2c) effectively removes all line broadening mechanisms other than spontaneous emission or pure relaxation processes (e.g., effects which enter only in the second term of the Liouville equation $\dot{\rho}=i[\rho, \mathscr{H}]-$ $\left.\mathscr{R}\left(\rho-\rho_{\text {eq }}\right)\right)$.

Figure 2 includes modifications in these long-established sequences to reflect the differences between optical and NMR spectroscopy. In particular, optical transitions often display long-range inhomogeneities which are greater than the available laser pulse jandwidth (Rabi frequency ${ }^{2}$ ), so only the central portion of the lineshape is properly refocused. A weak final pulse translates only this portion into excited state population. Taking a difference spectrum (final pulse of one phase minus final pulse phase shifted by $180^{\circ}$ ) samples only this portion, and also gives phase sensitive detection. 
a)
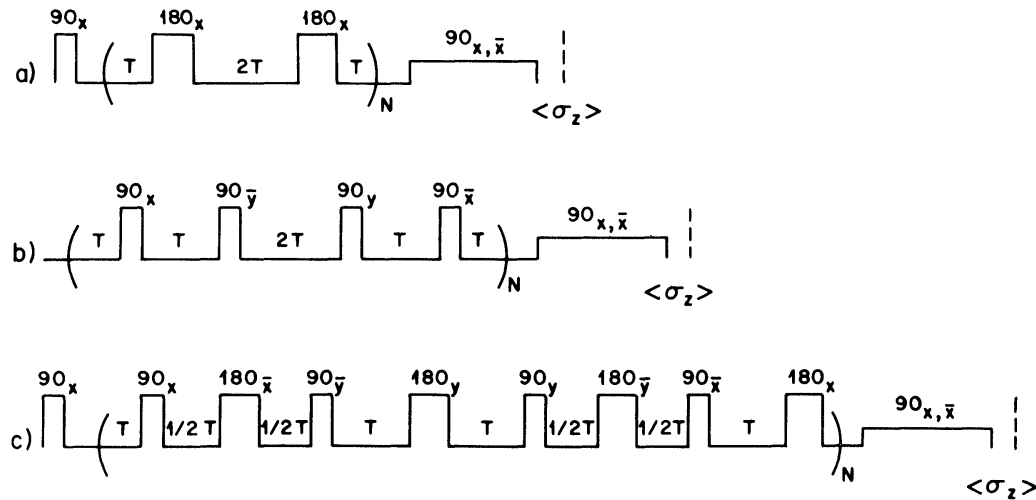

FIGURE 2 Line narrowing sequences for solid state electronic spectroscopy. The repeated portion of part (a) is a Carr-Purcell sequence, which refocuses both shortrange and long-range inhomogeneous broadening. Later improvements on this pulse sequence (different phases for the $180^{\circ}$ pulses) ${ }^{37}$ are also useful. The repeated portion of part (b) is a WAHUHA dipolar line narrowing sequence. Part (c) combines these sequences to eliminate essentially all reversible broadening mechanisms. In each part the fluorescence after the final weak pulse, minus the fluorescence produced when the final pulse is phase shifted by $180^{\circ}$, is the observed signal ZEWAWA sequence.

\section{MULTIPLE-QUANTUM AND MULTIPHOTON PROCESSES}

In conventional NMR the selection rule $\Delta m=1$ implies that only transitions corresponding to single spin flips are observable. The pulse sequence in Figure $3 \mathrm{a}$ breaks this selection rule. If $\tau \geqslant\left\langle D_{i j}^{-1}\right\rangle$ coherent superpositions between states with arbitrary values of $\Delta m$ (multiplequantum coherences) evolve during $t_{1}$. The primary advantage of this in NMR is spectral simplification. ${ }^{22,39}$

Since $D_{i j}$ terms also appear in the optical Hamiltonian (Eq. 5) multiple-quantum coherences can be induced in solid state optical spectroscopy. For example, a two-quantum coherence can be induced between the crystal ground state and states with two excited molecules. This coherence should be carefully distinguished from what would be called a double exciton in conventional optical spectroscopy. ${ }^{40}$ The incoherent excitation in the latter case implies limited phase correlation between the two excitons unless they collide; in the coherent case, however, the phase is always well defined. More importantly, multiple-quantum coherences will generally produce 
a)

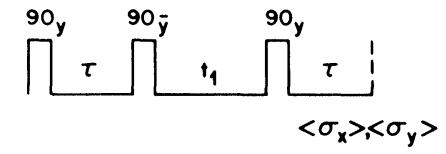

b)

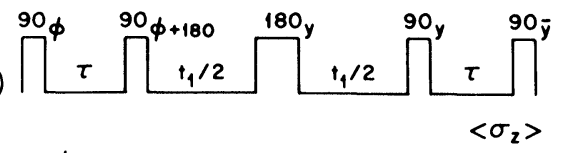

$\phi=\mathrm{Ct}_{1}$

FIGURE 3 Multiple-quantum pulse sequences. Part (a) is the simplest sequence which works in NMR. It produces coherences between states separated by an arbitrarily large number of spin flips (quanta absorbed). A $N$-quantum coherence decays as $\exp \left(-t_{1} / N T_{2}{ }^{*}\right)$, so the large inhomogeneous broadening in optical spectroscopy makes them unobservable. Part (b) incorporates modifications to echo these coherences and give phase sensitive detection. These modifications make optical multiple-quantum coherences observable. Since multiple-quantum coherences generate little polarization optical density effects are greatly reduced compared to absorption studies or photon echoes.

very little polarization (they do not correspond to allowed transitions) so optical density effects are reduced.

In fact a conventional echo sequence has been tried in mixed molecular crystals under the guise of the stimulated photon echo. ${ }^{13,14}$ Unfortunately it can be readily shown that an $N$-quantum coherence will decay $e^{-t_{1} / N T_{2}^{*}}$, i.e., $N$ times faster than the normal inhomogeneous broadening. ${ }^{20}$ Thus after a few psec only population decay $\left(e^{-t_{1} / T_{1}}\right)$ will be observed. This limitation can be circumvented by inserting an echoing $180^{\circ}$ pulse at $t_{1} / 2 .^{21}$ Phase sensitive detection ${ }^{7}$ is also essential for interpretable results, so a final pulse should be added and difference spectra measured (Figure 3b). Phase incrementation of the first two pulses $\left(\phi=C t_{1}\right)$ is then necessary to separate out the different coherences.

Multiphoton processes, such as pumping of vibrational local modes, can also be enhanced by multiple pulse trains. ${ }^{33}$ The normal limitation is bottlenecking effects due to energy mismatches in the first few transitions. These mismatches make it impossible to be near resonance for all transitions in the pathways to highly excited states. Phase modulated or amplitude modulated pulse sequences can dramatically enhance pumping efficiency. This enhancement can be qualitatively understood by noting that modulation with period $T$ produces sidebands with spacing $1 / T$ which can be made to simultaneously 
coincide with several transitions. The quantitative theory for two-level and three-level systems is derived in Ref. 33, where it is shown that enhancements of $10^{3}-10^{5}$ over nonmodulated pumping occur when the pulse lengths and delays are precisely correct.

\section{GAS PHASE ELECTRONIC TRANSITIONS- EXPERIMENTAL RESULTS}

The results of the last two sections show that phase coherent multiple pulse trains can be expected to be extremely valuable in optical spectroscopy. We have demonstrated that acousto-optic modulation ${ }^{7}$ of a single frequency laser generates sequences with independently controllable pulse amplitudes, delays, and phases. In essence, this technique generates a laser replica of any applied r.f. pulse sequence, limited only by the modulator risetime. The experimental setup at Caltech (Figure 4) produces pulses with up to $200 \mathrm{~mW}$ peak power and 4 ns risetimes.

We have used the technique for the suppression of spontaneous emission backgrounds by phase sensitive detection. Phase shifting the final pulse of a three-pulse echo sequence by $180^{\circ}$ inverts the coherent signal (the echo) without affecting the incoherent background, so a difference spectrum dramatically improves the accuracy of $T_{2}$ measurements. A $90^{\circ}$ phase shift would measure the induced polarization in quadrature with the normally observed component, which would be valuable for more complex sequences. ${ }^{32}$

Phase shifted rf pulses were also used to generate optical composite pulse trains. It has been known for many years in NMR that several pulses back-to-back ("composite pulses") can be less sensitive to inhomogeneities than is a single pulse. ${ }^{41}$ For example, r.f. inhomogeneities make it impossible for any single pulse to be a $\pi$ pulse throughout a real sample. A $\pi / 2$ pulse, followed immediately by a $\pi$ pulse $90^{\circ}$ out of phase, followed immediately by a $\pi / 2$ pulse of the original phase improves on this substantially, since it cancels the r.f. inhomogeneity to lowest order. ${ }^{41}$ There have also been some applications of composite pulse trains to compensate for transition inhomogeneity; this same pulse sequence creates large population inversions even far from resonance where the effects of a single pulse have substantially diminished. 


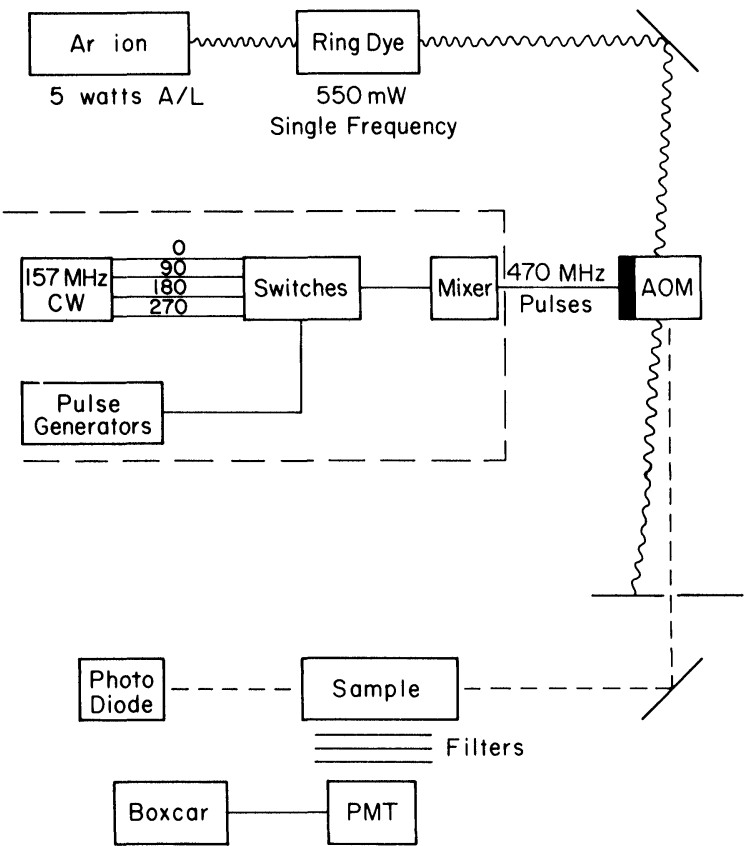

FIGURE 4 Experimental setup at Caltech. The acousto-optic modulator (AOM) diffracts and upconverts a single frequency ring dye laser when r.f. pulses at the acoustic phonon frequency $(470 \mathrm{Mhz})$ are applied. The dotted section of the apparatus is equivalent to a conventional NMR transmitter section. Off-resonance fluorescence is monitored perpendicular to the laser propagation direction.

Inhomogeneous broadening is much more severe in optical spectroscopy than it is in NMR, so that almost all optical experiments are dominated by inhomogeneous effects. Composite laser pulses are capable of turning over a larger fraction of the inhomogeneous profile far from resonance, thus effectively increasing the laser bandwidth and increasing the observable signal. Figure 5 shows that the fluorescence intensity after a single pulse (which was experimentally maximized if the pulse was $200 \mathrm{~ns}$ long) could be substantially increased by composite pulse trains. Figure 6 shows that echo intensities can also be increased. In favorable cases, these sequences will permit excitation of transitions over the entire inhomogeneous lineshape. 


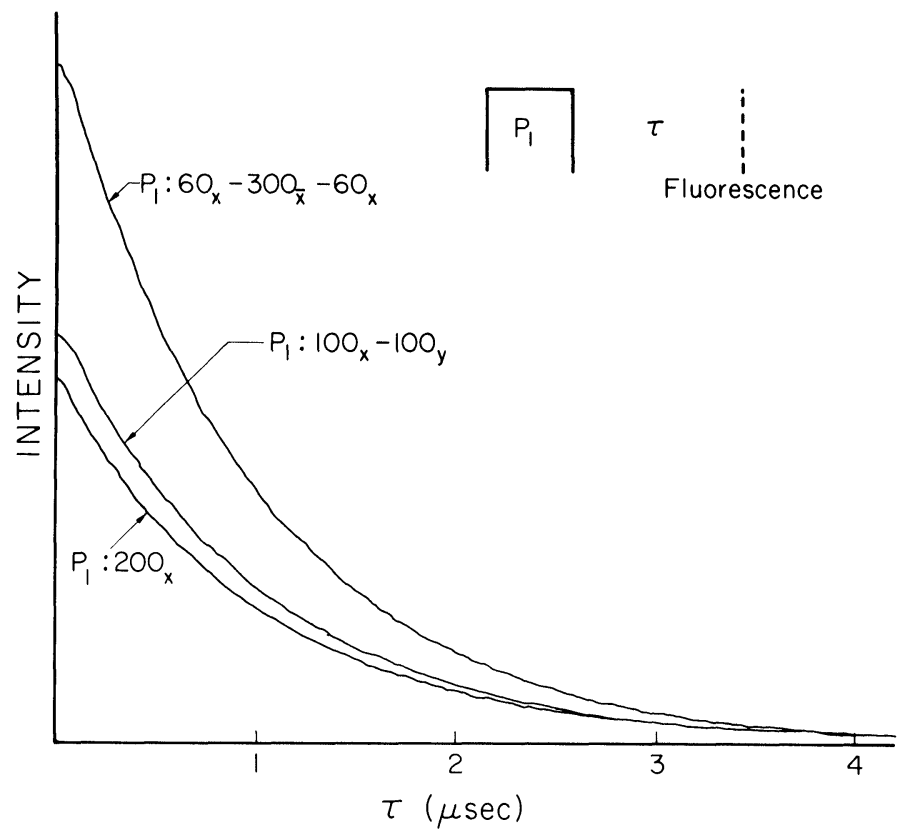

FIGURE 5 Composite pulses enhance fluorescence by exciting a larger fraction of the inhomogeneous lineshape. The maximum single pulse fluorescence was observed after a $200 \mathrm{~ns}$ pulse. Phase shifts increased the signal, and the experimental enhancements showed excellent agreement with theoretical calculations (see Ref. 30).

Finally, we showed that multiple pulse sequences can be used to measure previously inaccessible relaxation rates, such as the ground state population decay rate $T_{1 g}$ (Figure 7). The relative values of $T_{1 e}$ and $T_{1 \mathrm{~g}}$ for the case shown $\left(I_{2}\right.$ in the bulb at $\left.-12^{\circ}\right)$ imply that the excited state collisional cross section is substantially greater than the ground state cross section. Current experimental work includes extension of $T_{1 \mathrm{~g}}$ measurements to different temperatures and pressures of iodine or of buffer gases and complete characterization of the photon locking effect. ${ }^{42}$ The NMR spin locking sequence $90_{x}-T_{y}$, where the length $T$ of the second pulse is varied and $\left\langle I_{y}\right\rangle$ is measured immediately after that pulse, gives a signal which decays at a rate $T_{1 \rho}$ intermediate to $T_{2}$ and $T_{1}{ }^{43}$ Doppler broadening will make the direct optical analog of this sequence fail far from resonance, so polarization 


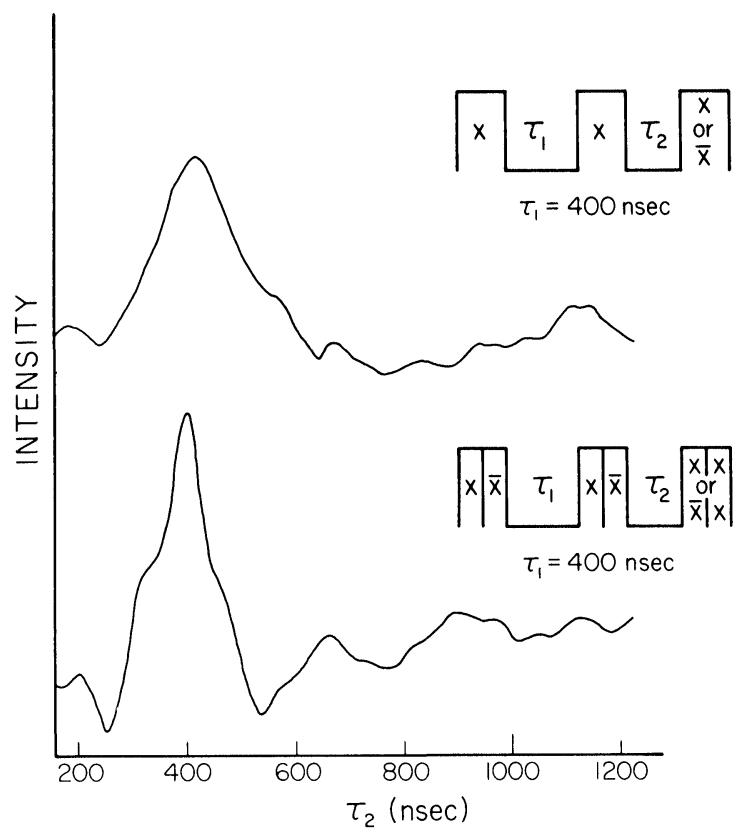

FIGURE 6 Composite pulses produce taller and sharper echoes, which would be expected if a larger fraction of the inhomogeneous lineshape is excited. The observed structure on the echo is theoretically expected (see Ref. 30 ).

detection would be ineffective. Phase sensitive detection near resonance (add in a final weak pulse, then take the difference after a $180^{\circ}$ phase shift) overcomes this problem to characterize the strength of collisional perturbations. Other work in progress includes lowtemperature mixed crystal studies, and extension of the technique to multiple frequency excitation, high peak powers, and picosecond resolution. Dual r.f. frequency generation permits high resolution pump-probe experiments of the gas phase velocity profile, with or without monitoring coherent effects. Higher power pump lasers and amplification schemes produce peak powers of many $\mathrm{kW}$ and 300 ps pulse lengths, with further gains expected. These higher powers greatly facilitate application of the pulse trains in Figures 2 and 3. 


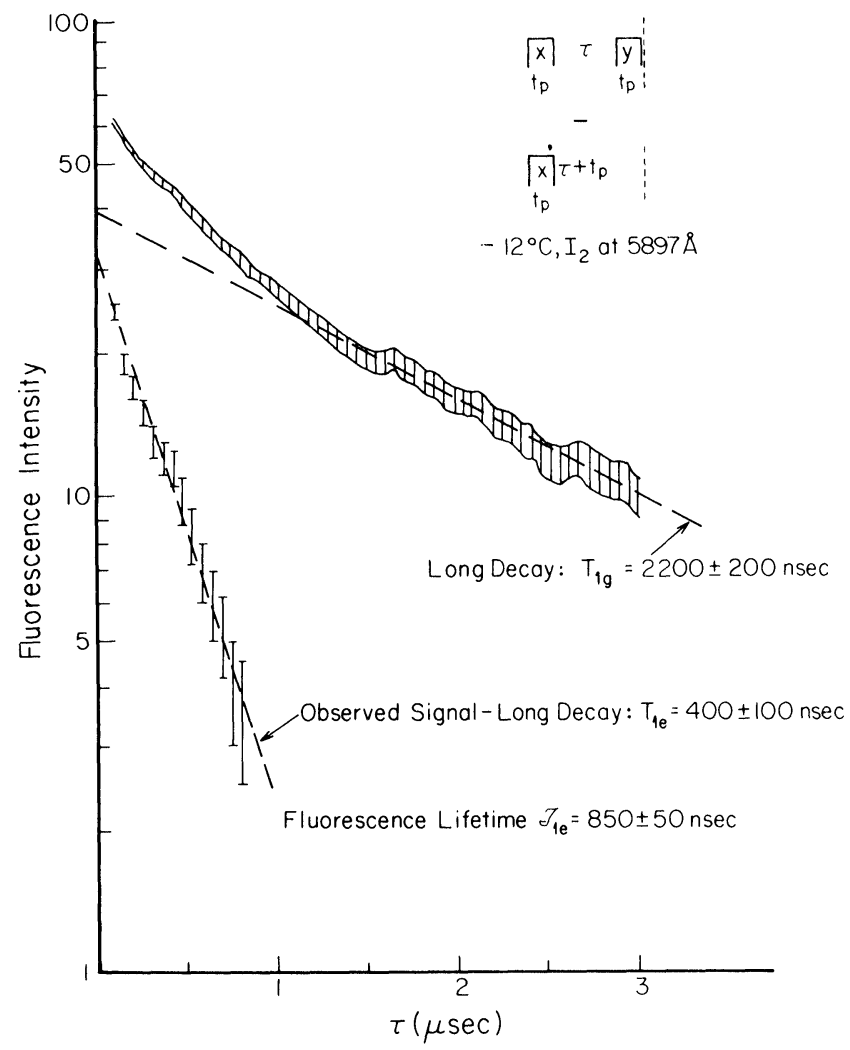

FIGURE 7 Measurement of ground state $\left(T_{1 \mathrm{~g}}\right)$ and excited state $\left(T_{1 e}\right)$ relaxation rates at $-12^{\circ}$ in $I_{2}$. The fluorescence decay for the sequence $\theta_{x}-\tau-\theta_{y}$, minus the fluorescence present if the last pulse is eliminated, should be biexponential with roughly equal coefficients for the two rates, as seen here. The implied collisional cross section is small for the ground state (see Ref. 30).

\section{CONCLUSIONS}

Phase coherent multiple pulse laser spectroscopy is theoretically valuable and experimentally possible. In fact, most of the novel NMR techniques developed over the last twenty years can now be used in laser spectroscopy. We expect that these techniques, along with the new sequences we are developing specifically for electronic 
interactions, will make coherent optical spectroscopy far more versatile and informative than it has been to date.

\section{Acknowledgements}

This research was supported by the National Science Foundation through Grant No. DMR81-05034 to Caltech. WSW was a National Science Foundation Postdoctoral Fellow at Caltech (1981). We are grateful to the National Science Foundation for making this work possible. AHZ would like to thank R. Silbey for inventing ZEWAWA.

\section{References}

1. F. Bloch, Phys. Rev. 70, 460 (1946); F. Bloch, W. W. Hansen and M. Packard, Phys. Rev. 70, 474 (1946); E. M. Purcell, H. C. Torrey and R. Pound, Phys. Rev. 69, 37 (1946).

2. I. I. Rabi, N. F. Ramsey and J. Schwinger, Ref. Mod. Phys. 26, 157 (1964).

3. A. Abragam, The Principles of Nuclear Magnetism (Oxford, London, 1963).

4. T. C. Farrar and E. D. Becker, Pulse and Fourier Transform NMR (Academic, New York, 1971).

5. R. H. Dicke, Phys. Rev. 93, 99 (1954).

6. R. P. Feynman, F. L. Vernon and R. W. Hellwarth, J. Appl. Phys. 28, 49 (1957).

7. A. H. Zewail, T. E. Orlowski, K. E. Jones and D. E. Godar, Chem. Phys. Lett. 48, 256 (1977); T. E. Orlowski, K. Jones and A. H. Zewail, Chem. Phys. Lett. 54, 197 (1978).

8. D. E. Copper, R. W. Olson and M. D. Fayer, Chem. Phys. Lett. 52, 399 (1977).

9. H. de Vries, P. DeBree and D. A. Wiersma, Chem. Phys. Lett. 52, 399 (1977).

10. R. G. Brewer and R. L. Schoemaker, Phys. Rev. Lett. 27, 631 (1971); R. G. Brewer and A. Z. Genack, Phys. Rev. Lett. 36, 959 (1976).

11. P. R. Berman, T. W. Mossberg and S. R. Hartmann, Phys. Rev. A 25, 2550 (1982); R. Kachyu, T. H. Chen, T. W. Mossberg and S. R. Hartmann, Phys. Rev. A25, 1546 (1982).

12. A. H. Zewail, Acc. Chem. Res. 13, 360 (1980) and references therein.

13. D. E. Cooper, R. W. Olson and M. D. Fayer, J. Chem. Phys. 72, 2332 (1980); R. W. Olson, H. W. H. Lee, F. G. Patterson and M. D. Fayer, J. Chem. Phys. 76, 31 (1982).

14. J. B. W. Morsink, B. Kruizinga and D. A. Wiersma, Chem. Phys. Lett. 76, 218 (1980).

15. S. L. McCall and E. H. Hahn, Phys. Rev. Lett. 18, 908 (1967).

16. J. G. Powles and P. Mansfield, Phys. Lett. 2, 58 (1962); P. Mansfield, Phys. Rev. 137, 1962 (1965).

17. J. S. Waugh, L. M. Huber and U. Haeberlen, Phys. Rev. Lett. 20, 180 (1968); U. Haeberlen, Advances in Magnetic Resonance, (Academic, New York, 1976) Suppl. 1.

18. A. Wokaun and R. R. Ernst, Molec. Phys. 36, 317 (1978).

19. M. E. Stoll, A. J. Vega and R. W. Vaughn, J. Chem. Phys. 67, 2029 (1977).

20. S. Vega, T. W. Shattuck and A. Pines, Phys. Rev. Lett. 37, 43 (1976).

21. G. Drobny, A. Pines, S. Sinton, D. P. Weitekamp and D. Wemmer, Faraday Div. Chem. Soc. Symp. 13, 49 (1979). 
22. G. Bodenhausen, R. L. Vold and R. R. Vold, J. Magn. Reson. 37, 93 (1980); A. Wokaun and R. R. Ernst, Chem. Phys. Lett. 52, 407 (1977); H. Hatanaka, T. Terao and T. Hashi, J. Phys. Soc. Jpn. 39, 835 (1975).

23. For a recent review see G. Bodenhausen, Prog. NMR Spect. 14, 113 (1981).

24. W. S. Warren, S. Sinton, D. P. Weitekamp and A. Pines, Phys. Rev. Lett. 43, 1791 (1979).

25. W. S. Warren, D. P. Weitekamp and A. Pines, J. Chem. Phys. 73, 2084 (1981); W. S. Warren and A. Pines, J. Chem. Phys. 74, 2808 (1981).

26. W. S. Warren and A. Pines, Chem. Phys. Lett. 88, 441 (1982).

27. R. Freeman and G. A. Morris, Bull. Mag. Res. 1, 18 (1979).

28. K. Wuthrich, Macromol. Chem. Phys., Suppl. 5, 234-252 (1981) and references therein.

29. W. S. Warren and A. H. Zewail, J. Chem. Phys. 75, 5956 (1981).

30. W. S. Warren and A. H. Zewail, J. Chem. Phys. 78, 2279 (1983).

31. W. S. Warren and A. H. Zewail, J. Phys. Chem. 85, 2309 (1981).

32. W. S. Warren and A. H. Zewail, J. Chem. Phys. 78, 2298 (1983).

33. W. S. Warren and A. H. Zewail, J. Chem. Phys. 78, 3583 (1983).

34. J. W. Emsley and J. C. Lindon, NMR Spectroscopy Using Liquid Crystal Solvents (Pergamon, Oxford, 1975); P. Diehl and C. L. Khetrapal, NMR: Basic Principles and Progress, Vol. 1 (1971).

35. H. Port, University of Stuttgart, unpublished results.

36. D. M. Burland, J. Chem. Phys. 59, 4383 (1973); D. M. Burland and R. M. MacFarlane, J. Luminesc. 12-13, 213 (1976); D. M. Burland, U. Konzelmann and R. M. MacFarlane, J. Chem. Phys. 67, 1926 (1977); R. M. MacFarlane, U. Konzelmann and D. M. Burland, J. Chem. Phys. 65, 1022 (1976).

37. S. Meiboom and G. Gill, Rev. Sci. Instrum. 29, (1958); H. Y. Carr and E. M. Purcell, Phys. Rev. 94, 630 (1954).

38. J. Schmidt, P. Berman and R. Brewer, Phys. Rev. Lett. 31, 1103 (1973).

39. W. S. Warren and A. Pines, J. Am. Chem. Soc. 103, 1613 (1981).

40. A. Davydov, Theory of Molecular Excitons (Plenum, New York, 1971).

41. R. Freeman, S. P. Kempsell and M. H. Levitt, J. Mag. Reson. 38, 453 (1980); M. H. Levitt and R. Freeman, J. Mag. Reson. 43, 502 (1981); M. H. Levitt, R. Freeman and T. Frenkel, J. Mag. Reson. 47, 328 (1982).

42. E. Sleva, W. S. Warren and A. H. Zewail, to be published.

43. I. Solomon, C. R. Acad. Sci. Paris 248, 92 (1959). 